\title{
H-repair in supravalvular aortic stenosis
}

\author{
Shuhua Luo, MD, PhD, ${ }^{\text {a,b,c }}$ Christoph Haller, MD, ${ }^{\mathrm{a}, \mathrm{b}}$ Mimi Xiaoming Deng, $\mathrm{BScH},{ }^{\mathrm{a}, \mathrm{b}}$ and \\ Osami Honjo, MD, PhD, ${ }^{a, b}$ Toronto, Ontario, Canada, and Chengdu, China
}

\footnotetext{
From the a Division of Cardiovascular Surgery, Labatt Family Heart Centre, The Hospital for Sick Children, Toronto, Ontario, Canada; ${ }^{b}$ Department of Surgery, University of Toronto, Toronto, Ontario, Canada; and ${ }^{\mathrm{c} D e p a r t-}$ ment of Cardiovascular Surgery, West China Hospital of Sichuan University, Chengdu, China.

Disclosures: The authors reported no conflicts of interest.

The Journal policy requires editors and reviewers to disclose conflicts of interest and to decline handling or reviewing manuscripts for which they may have a conflict of interest. The editors and reviewers of this article have no conflicts of interest.

Received for publication Dec 17, 2020; accepted for publication Dec 21, 2020; available ahead of print Jan 6, 2021.

Address for reprints: Osami Honjo, MD, PhD, Division of Cardiovascular Surgery, Labatt Family Heart Centre, The Hospital for Sick Children, 555 University Ave, Toronto, ON, Canada M5G1X8 (E-mail: osami.honjo@ sickkids.ca).

JTCVS Techniques 2021;6:114-7

2666-2507

Copyright (c) 2021 The Authors. Published by Elsevier Inc. on behalf of The American Association for Thoracic Surgery. This is an open access article under the CC BY-NC-ND license (http://creativecommons.org/licenses/bync-nd/4.0/).

https://doi.org/10.1016/j.xjtc.2020.12.003
}

$\rightarrow$ Video clip is available online.

Multisinus aortoplasty (2- or 3-sinus patch) restores normal hemodynamics and reduces the need for reoperation compared with the classic 1-patch technique for supravalvular aortic stenosis (SAS) repair. ${ }^{1-3}$ Since January 2000, 9 out of 33 patients $(27.3 \%)$ who underwent SAS repair in our institution using an inverted Y-shaped patch demonstrated echocardiographic evidence of impaired coronary blood flow and hemodynamic instability due to right coronary artery (RCA) distortion and kinking. Here, we modified the bifurcate patch with 2 separate vertical patches (H-repair) to maintain the RCA geometry and avoid compromising coronary artery flow.

\section{SURGICAL TECHNIQUE}

After a median sternotomy, the pericardium is harvested and treated with a glutaraldehyde solution for 5 minutes. Cardiopulmonary bypass is initiated via aortic and right atrial cannulation if there is no interatrial communication. We prefer to cannulate the proximal aortic arch to gain enough space for aortic root reconstruction. The heart is arrested by antegrade cardioplegia. A transverse incision is made on the ascending aorta approximately $5 \mathrm{~mm}$ above the sinotubular junction (STJ). A vertical incision is then made from the right

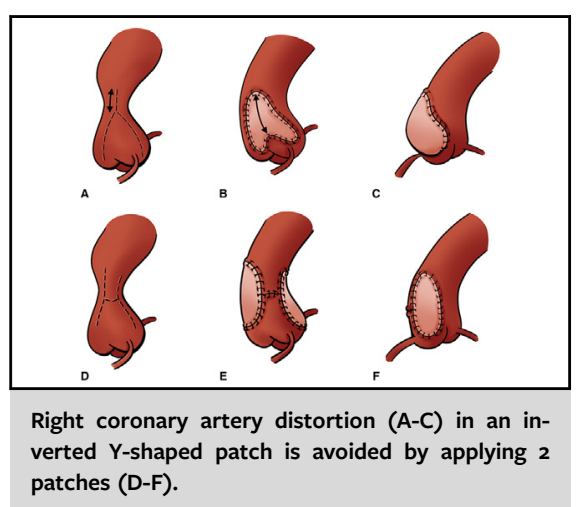

\begin{abstract}
CENTRAL MESSAGE
The right coronary artery

distortion associated with in-

verted $Y$-shaped patch repair of

SAS, is avoided in H-repair by

using 2 separate vertical patches

to reconstruct the aortic root.
\end{abstract}

See Commentaries on pages 118 and 120.

end of the transverse incision into the middle of the noncoronary sinus. Another similar vertical incision is curved from the left end of the transverse incision into the right coronary sinus, close to the right and left coronary cusp commissure. These 2 vertical incisions are extended superiorly into the distal ascending aorta, forming an H-shaped incision.

Two separate rectangle-shaped pericardium patches are then used to reconstruct the noncoronary and right coronary cusps. The proximal ends of the patches are trimmed according to the circumference of the corresponding aortic annulus, with care taken to not oversize the right coronary sinus patch. The sizes of distal ends of the patches are usually equal and calculated based on the age-normalized circumference of the STJ. The coronary cusp patches are extended above the transverse incision to reconstruct the ascending aorta along the 2 vertical incisions. The initial transverse incision is primarily reapproximated to maintain the original longitudinal dimension of the ascending aorta (Figure 1, $A-D$, Video 1).

For patients with a diffusely small arch, the concomitant arch reconstruction is performed under deep hypothermic 


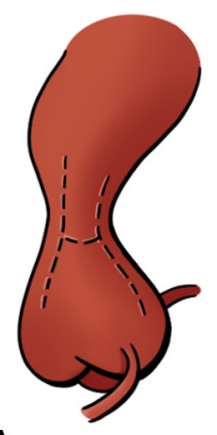

A

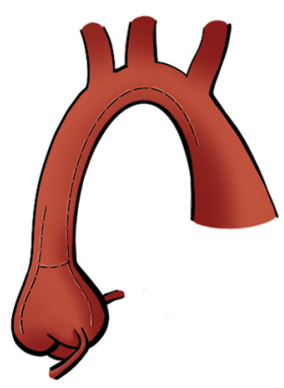

E

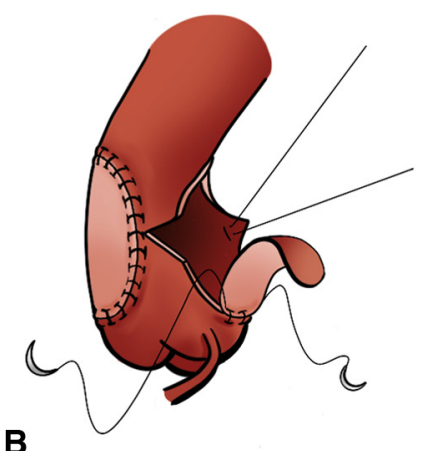

B

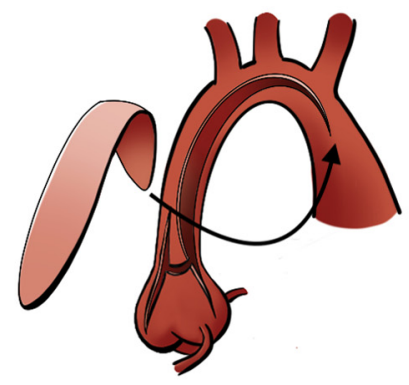

$\mathbf{F}$

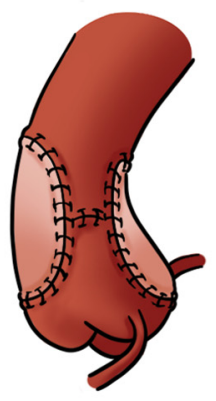

C

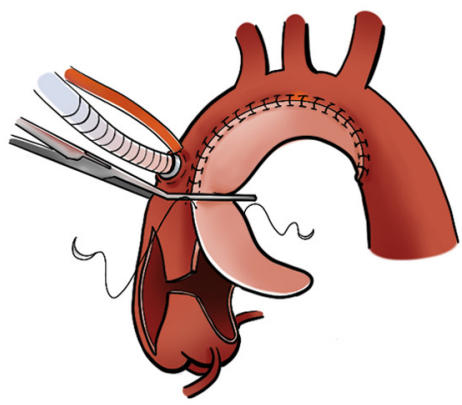

G

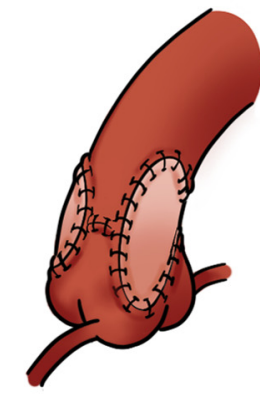

D

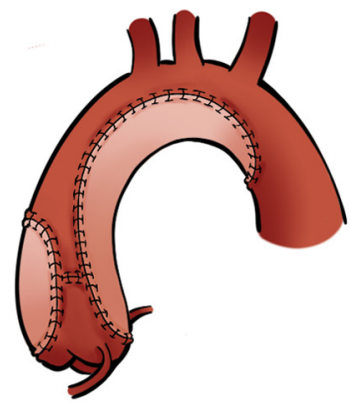

$\mathbf{H}$

FIGURE 1. H-repair in patients with supravalvular aortic stenosis. A, A transverse incision is made on the ascending aorta approximately 5 mm above the sinotubular junction (STJ). A vertical incision is then made from the right end of the transverse incision into the middle of the noncoronary sinus. A similar vertical incision is curved from the left end of the transverse incision into the right coronary sinus close to the right and left coronary cusp commissure. These 2 vertical incisions are extended superiorly into the distal ascending aorta, forming an $H$-shaped incision. B, Two separate long oval-shaped pericardial patches tailored according to direct measurements of the STJ and aortic annulus are then used to reconstruct the noncoronary and right coronary cusp. $\mathrm{C}$ and $\mathrm{D}$, The coronary cusp patches are extended above the transverse incision to reconstruct the ascending aorta along the two vertical incisions. The initial transverse incision is primarily reapproximated to maintain the original longitudinal dimension of ascending aorta. E, For patients with a diffusely small arch, the concomitant arch reconstruction is performed under deep hypothermic circulatory arrest. The right coronary sinus incision is extended into the arch beyond the ligamentum. F, A banana-shaped pericardial patch is used to reconstruct the entire arch under selective cerebral perfusion. G, When the patch comes down to the ascending aorta, the full bypass is resumed, and the cross-clamp is reapplied. $\mathrm{H}$, The right coronary sinus is reconstructed with the same patch used to repair the aortic arch. A separately treated pericardial patch is used to enlarge the noncoronary sinus.

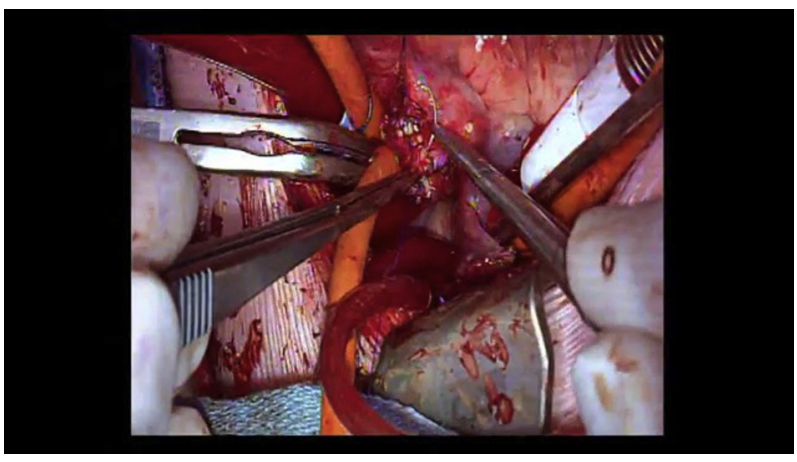

VIDEO 1. H-repair in a 12-month-old boy diagnosed with supravalvular aortic stenosis. Follow-up transthoracic echocardiography at 3 months showed an unobstructed aortic root and sinotubular junction without flow acceleration, good biventricular function, and patent left and right coronary arteries. circulatory arrest (DHCA). An H-shaped incision is made on the ascending aorta as described above. After DHCA is initiated and the cross-clamp is removed, the right coronary sinus incision is extended into the arch beyond the ligamentum. A banana-shaped pericardium is used to reconstruct the entire arch under selective cerebral perfusion. When the patch comes down to the ascending aorta, the full bypass is resumed, and the cross-clamp is reapplied. The right coronary sinus is reconstructed with the same patch used to repair the aortic arch. A separately treated pericardium patch is used to enlarge the noncoronary sinus (Figure 1, E-H).

\section{DISCUSSION}

Since January 2014, a total of 8 SAS patients underwent H-repair. The requirement for patient consent was waived for this retrospective technique report. The median patient 

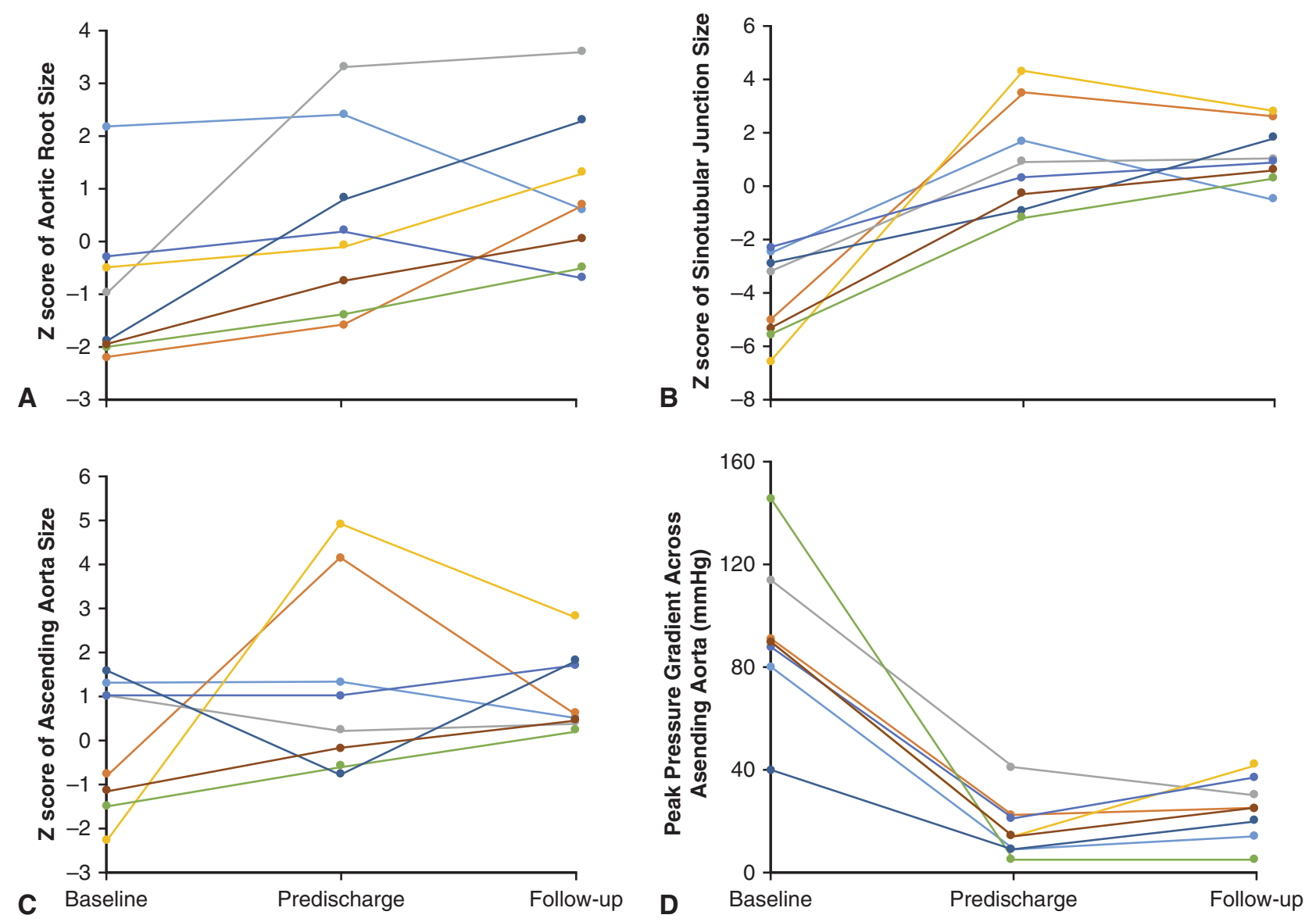

FIGURE 2. The change in aortic structure dimensions before and after H-repair. A-C, The $z$-scores of anatomic parameters including the aortic root (A), sinotubular junction (B), and ascending aorta (C) increased to normal ranges at predischarge and remained stable during medium-term (median, 2.6 years; range, 1.4-3.9 years) follow-up, as shown on echocardiography of 8 patients who underwent H-repair. D, The peak pressure gradient across the ascending aorta was significantly decreased at predischarge and follow-up.

age was 12.4 months (range, 8.0-78.0 months), and median weight was $7.6 \mathrm{~kg}$ (range, $6.0-18.4 \mathrm{~kg}$ ) at the time of operation. The median Cardiopulmonary bypass time was 106 minutes (range 89.7-124.3 minutes), and median cross-clamp time was 81.5 minutes (range, 74.5-94.5 minutes) for 5 patients with narrowing at the level of STJ. Another 3 patients underwent concomitant arch reconstruction, with DHCA times of 5, 11 and, 24 minutes, respectively. The results at the medium-term follow-up (median, 2.6 years; range, 1.4-3.9 years) were favorable (Figure 2). There were no deaths, and 1 patient experienced severe aortic insufficiency owing to right coronary cusp prolapse resulting from previous aortic valve commissurotomy for a unicuspidal stenotic valve and secondary mitral insufficiency at 3 months postrepair.

Based on our observations, RCA distortion and kinking are usually caused by the bulging of the adjacent redundant longitudinal dimension of an inverted Y-shaped patch, which shifts the proximal RCA anteriorly, inferiorly, and laterally (Figure 3, $A-C$ ). Shortening the longitudinal length of the patch with plication immediately reduces the kinking of the proximal RCA, highlighting the importance of maintaining the original length of ascending aorta. This experience inspired the idea of H-repair. The noncoronary and right coronary sinus are precisely and effectively augmented by 2 separately tailored vertical patches. which also maintains the longitudinal geometry by primary reapproximation of the transverse incision to avoid RCA distortion/kinking (Figure 3, D-F).

Given that the sinuses are usually asymmetrically affected, H-repair is an attractive alternative in patients with a relatively large left sinus not necessitating 3-sinus patch augmentation, as long as left coronary artery ostial patency is secured by adequate resection of a potential ostial or supraostial ridge. In addition, H-repair significantly simplifies concomitant arch repair by easily extending the vertical right coronary cusp patch to reconstruct the arch. 


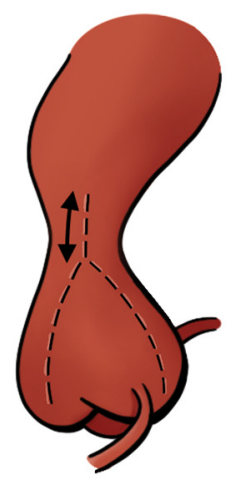

A

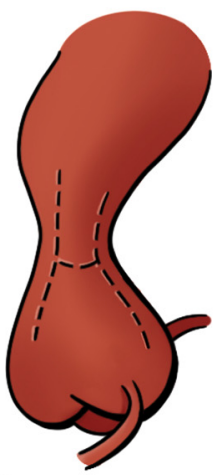

D

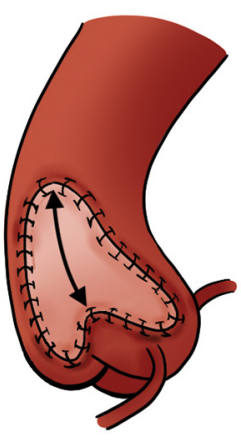

B

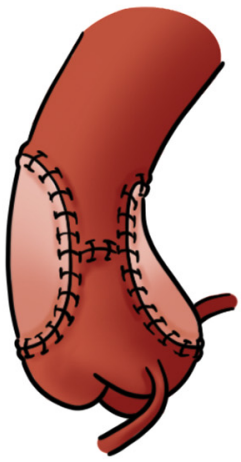

$\mathbf{E}$

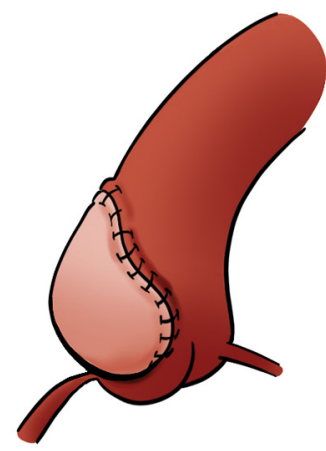

C

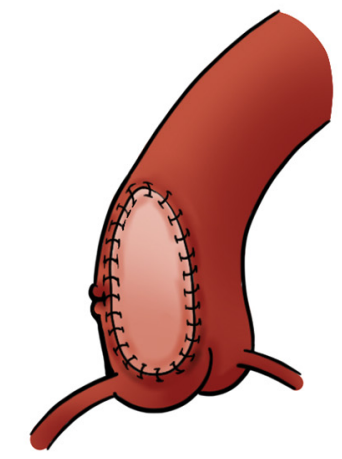

$\mathbf{F}$

FIGURE 3. Right coronary artery distortion (A-C) in an inverted Y-shaped patch is avoided by applying 2 patches (D-F).

Further follow-up is warranted to assess long-term outcomes of H-repair.

\section{References}

1. Fricke TA, d'Udekem Y, Brizard CP, Wheaton G, Weintraub RG, Konstantinov IE. Surgical repair of supravalvular aortic stenosis in children with Williams syndrome: a 30-year experience. Ann Thorac Surg. 2015;99:1335-41.
2. Kramer P, Absi D, Hetzer R, Photiadis J, Berger F, Alexi-Meskishvili V. Outcome of surgical correction of congenital supravalvular aortic stenosis with two- and three-sinus reconstruction techniques. Ann Thorac Surg. 2014;97:634-40.

3. Kaushal S, Backer CL, Patel S, Gossett JG, Mavroudis C. Midterm outcomes in supravalvular aortic stenosis demonstrate the superiority of multisinus aortoplasty. Ann Thorac Surg. 2010;89:1371-7. 